\title{
Synthesis of a pH-Responsive Functional Covalent Organic Frame- work via Facile and Rapid One-Step Postsynthetic Modification and Its Application in Highly Efficient $\boldsymbol{N}^{1}$-Methyladenosine Extraction
}

Yu-Fang Ma, Fang Yuan, Yue Yu, Ying-Lin Zhou* and Xin-Xiang Zhang*

Beijing National Laboratory for Molecular Sciences (BNLMS), MOE Key Laboratory of Bioorganic Chemistry and Molecular Engineering, College of Chemistry, Peking University, Beijing 100871, China

*Corresponding authors' contact information

E-mail: zhouyl@pku.edu.cn; Fax: +86-10-62754112; Tel: +86-10-62754112.

E-mail: zxx@pku.edu.cn; Fax: +86-10-62754112; Tel: +86-10-62754112.

\section{Contents}

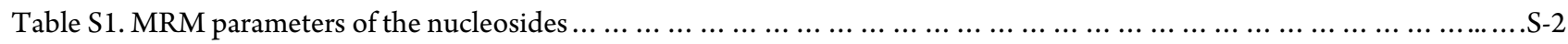

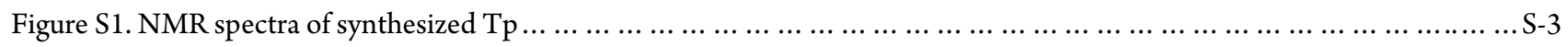

Figure S2. FT-IR spectra of synthesized TpPa-1@Au@GSH, synthesized TpPa-1, synthesized Tp and Pa-1 ................

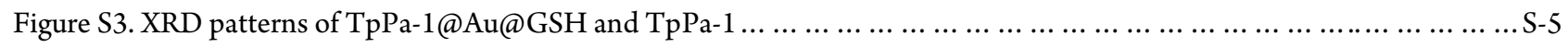

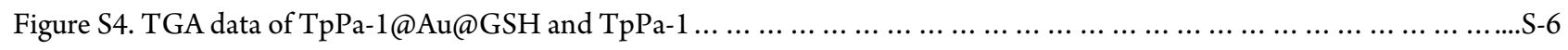

Figure S5. ${ }^{13} \mathrm{C}$ CP-TOSS MAS solid-state NMR spectra of TpPa-1 and TpPa-1@Au@GSH........................................

Figure S6. Performance of $\mathrm{m}^{1} \mathrm{~A}$ extraction by TpPa-1@Au@GSH with different composition of the loading buffer as well as washing buffer. S-8

Figure S7. Performance of $\mathrm{m}^{1} \mathrm{~A}$ adsorption by TpPa-1@Au@GSH after being reused for several times... S-9

Figure S8. Performance of $\mathrm{m}^{1} \mathrm{~A}$ adsorption by TpPa-1@Au@GSH storing for different period of time at room temperature S-10

Figure S9. LC-MS chromatographs of Arabidopsis thaliana roots without extraction and with extraction using TpPa1@Au@GSH. S-11 
Figure S10. LC-MS chromatographs of mouse serum without extraction and with extraction using TpPa-1@Au@GSH...S-12

Table S1. MRM parameters of the nucleosides

\begin{tabular}{cccc}
\hline Nucleoside & Q1 & Q3 & Collision energy $(\mathrm{eV})$ \\
\hline $\mathrm{A}$ & 268.2 & 136.1 & 26.00 \\
$\mathrm{~m}^{1} \mathrm{~A}$ & 282.1 & 150.1 & 26.00 \\
$\mathrm{~m}^{6} \mathrm{~A}$ & 282.0 & 150.1 & 24.00 \\
$\mathrm{Am}$ & 282.0 & 136.1 & 23.00 \\
$\mathrm{I}$ & 269.1 & 137.1 & 21.00 \\
$\mathrm{G}$ & 284.1 & 152.2 & 23.33 \\
$\mathrm{Gm}$ & 298.1 & 152.1 & 21.00 \\
$\mathrm{U}$ & 245.1 & 113.0 & 19.00 \\
$\mathrm{Um}$ & 258.9 & 113.1 & 20.00 \\
$\Psi$ & 245.1 & 179.1 & 14.72 \\
$\mathrm{C}$ & 244.1 & 112.1 & 24.00 \\
$\mathrm{Cm}$ & 258.0 & 112.0 & 20.00 \\
$\mathrm{~m}^{5} \mathrm{C}$ & 258.0 & 126.1 & 27.16 \\
$\mathrm{hm}$ & 274.0 & 142.1 & 16.00 \\
\hline
\end{tabular}



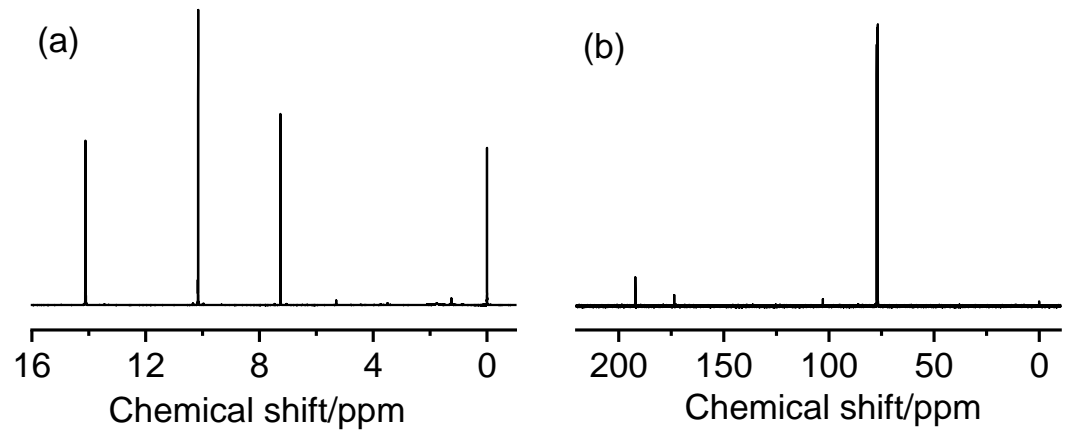

Figure S1. (a) ${ }^{1} \mathrm{H}$ NMR and (b) ${ }^{13} \mathrm{C}$ NMR spectra of synthesized Tp. 


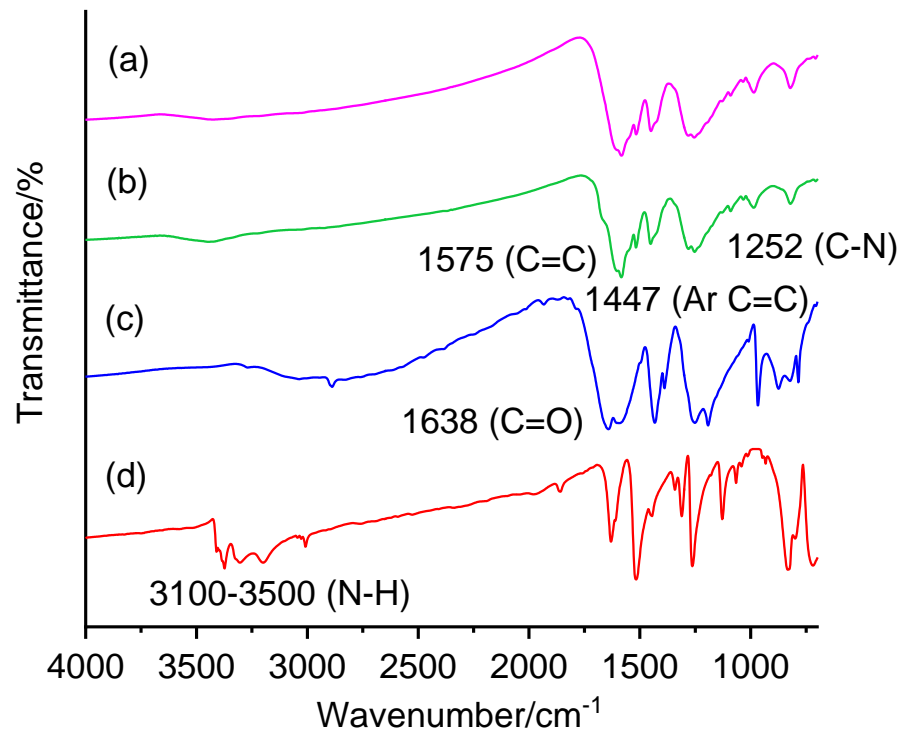

Figure S2. FT-IR spectra of (a) synthesized TpPa-1@Au@GSH, (b) synthesized TpPa-1, (c) synthesized Tp and (d) Pa-1. 


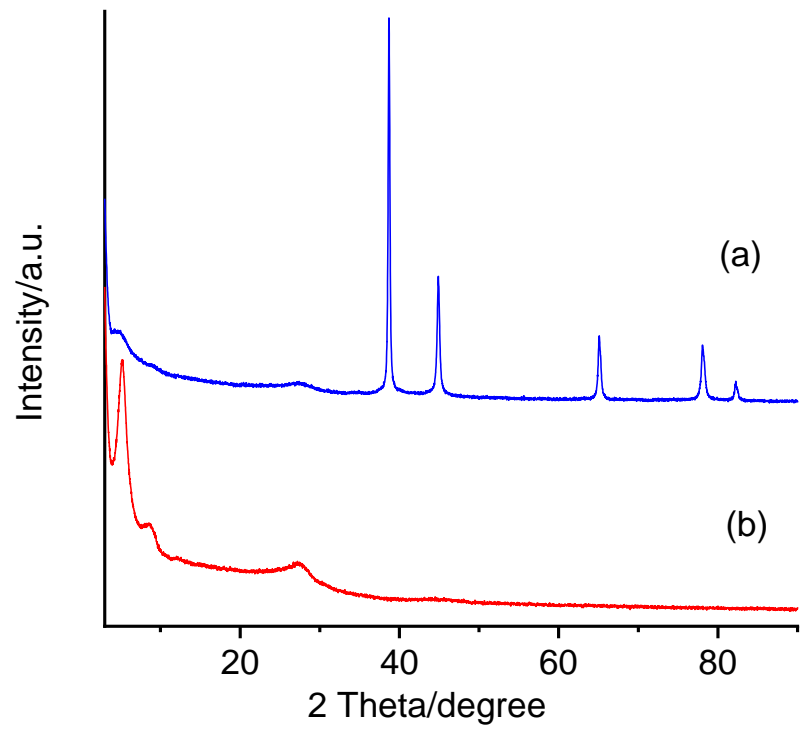

Figure S3. XRD patterns of (a) TpPa-1@Au@GSH and (b) TpPa-1. 


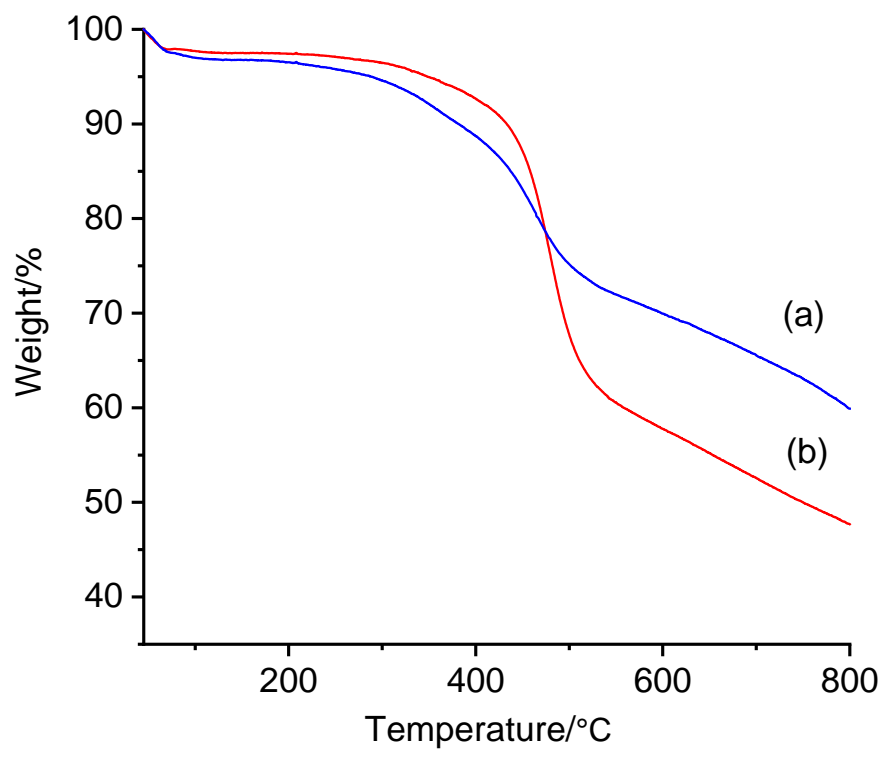

Figure S4. TGA data of (a) TpPa-1@Au@GSH and (b) TpPa-1. 

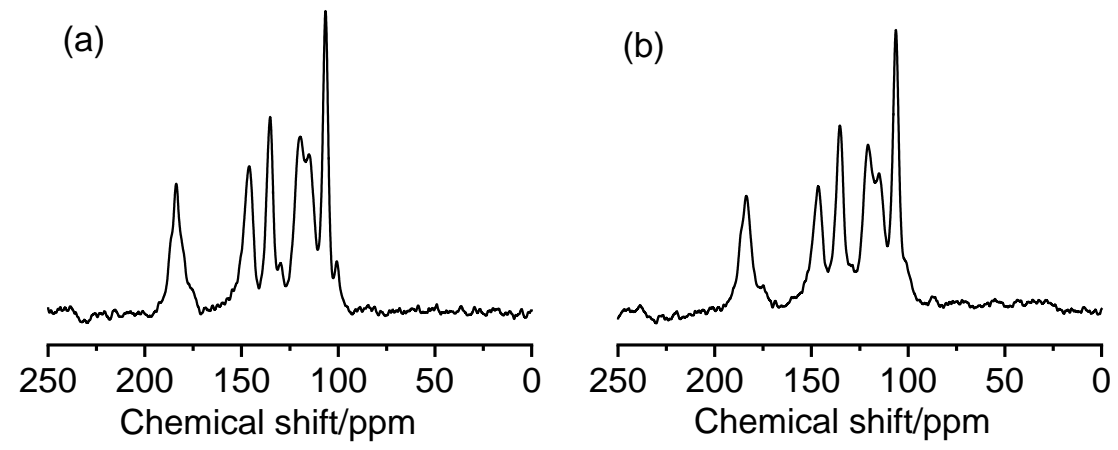

Figure S5. ${ }^{13} \mathrm{C}$ CP-TOSS MAS solid-state NMR spectra of (a) TpPa-1 and (b) TpPa-1@Au@GSH. 


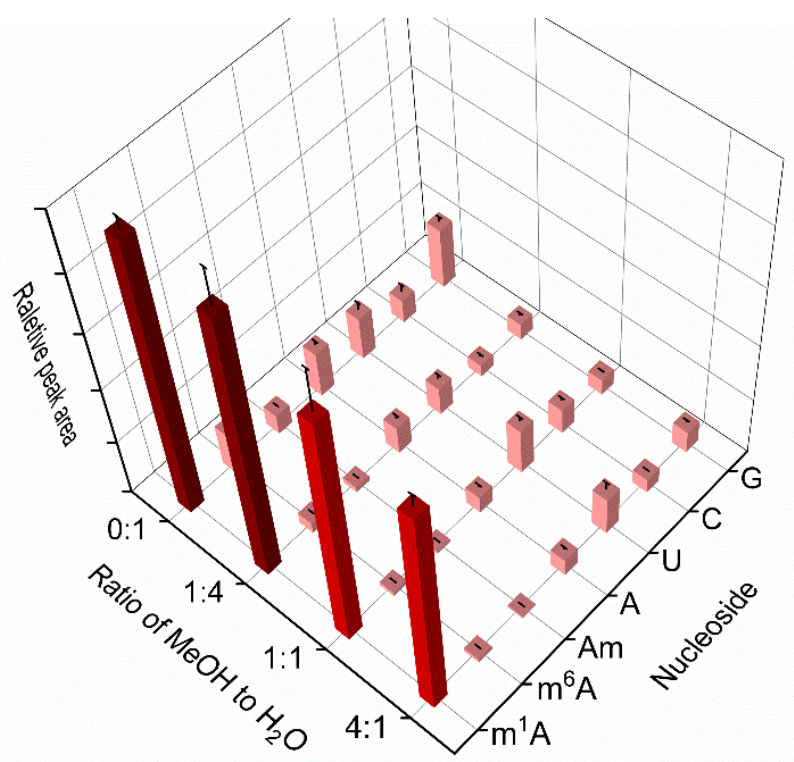

Figure S6. Performance of $\mathrm{m}^{1} \mathrm{~A}$ extraction by TpPa-1@Au@GSH with different composition of the loading buffer as well as washing buffer. $\mathrm{MeOH} / \mathrm{H}_{2} \mathrm{O}(\mathrm{v} / \mathrm{v})=0 / 1,1 / 4,1 / 1$ and 4/1. For each nucleoside, the $z$ axis=peak area of detected nucleoside/peak area of this nucleoside before extraction. Color from deep to light represents the number from large to small. The error bars represent the standard deviations of three parallel tests. 


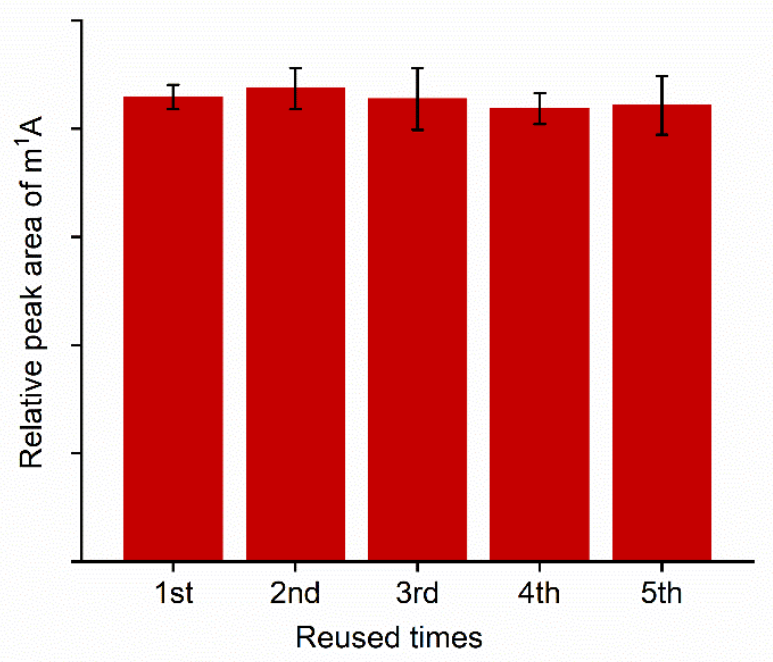

Figure S7. Performance of $\mathrm{m}^{1} \mathrm{~A}$ adsorption by TpPa-1@Au@GSH after being reused for several times. The $y$ axis represents the peak area of $\mathrm{m}^{1} \mathrm{~A}$ compared with other groups. The error bars represent the standard deviations of three parallel tests. 


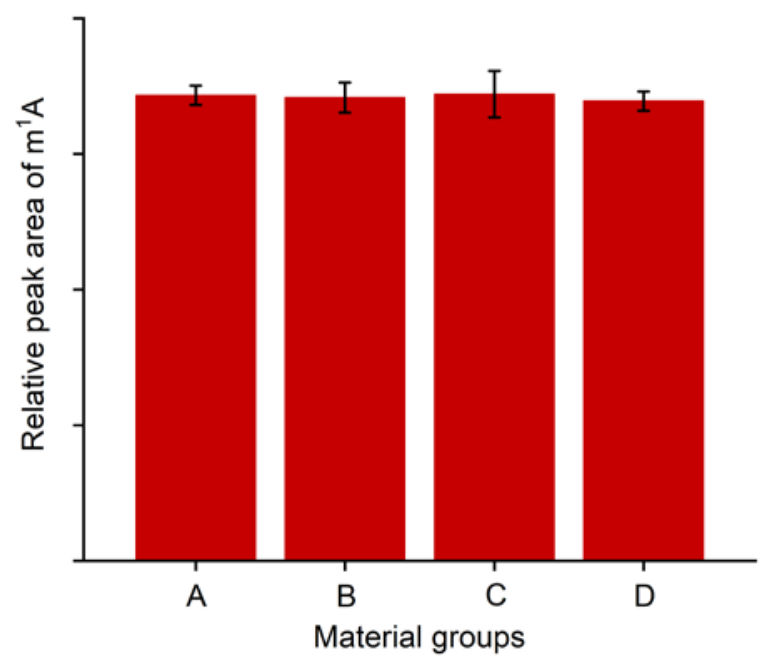

Figure S8. Performance of $\mathrm{m}^{1} \mathrm{~A}$ adsorption by TpPa-1@Au@GSH storing for different period of time at room temperature. The $x$ axis represents the materials that (A) freshly synthesized, (B) stored for 5 months, (C) stored for 6 months and (D) stored for 8 months. The $y$ axis represents the peak area of $\mathrm{m}^{1} \mathrm{~A}$ compared with other groups. The error bars represent the standard deviations of three parallel tests. 

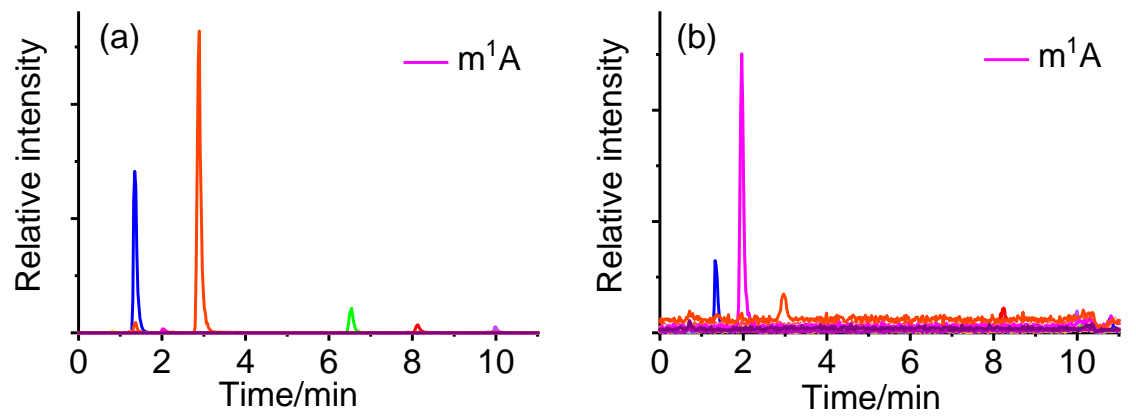

Figure S9. LC-MS chromatographs of Arabidopsis thaliana roots (a) without extraction and (b) with extraction using TpPa-1@Au@GSH. 

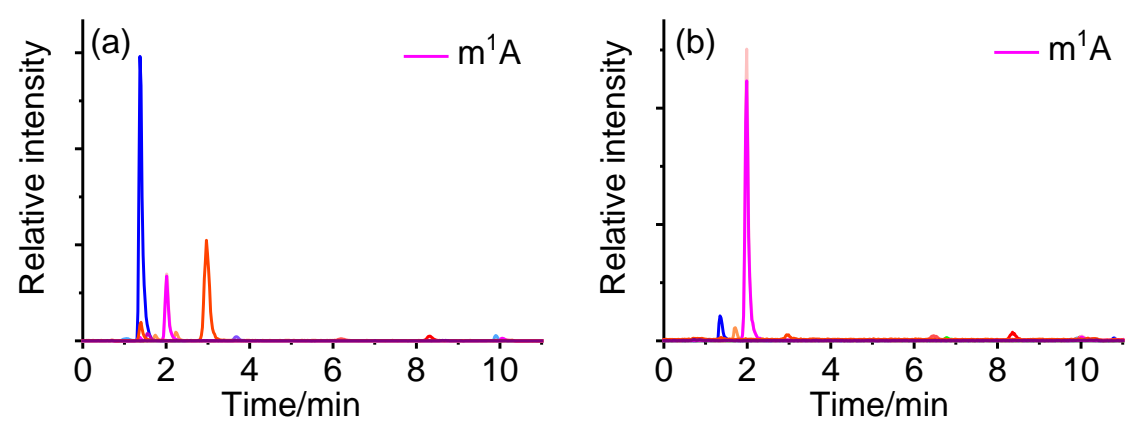

Figure S10. LC-MS chromatographs of mouse serum (a) without extraction and (b) with extraction using TpPa-1@Au@GSH. 